\title{
MR Image Analytics to Characterize Upper Airway Architecture in Children with OSAS
}

\author{
Yubing Tong ${ }^{\mathrm{a}}$, Jayaram K. Udupa ${ }^{* a}$, Drew A. Torigian ${ }^{\mathrm{a}}$, Monica M. S. Matsumoto ${ }^{\mathrm{a}}$, Sanghun $\operatorname{Sin}^{\mathrm{b}}$, \\ Raanan Arens ${ }^{\mathrm{b}}$ \\ ${ }^{a}$ Medical Image Processing Group, 423 Guardian Drive, 4th Floor Blockley Hall, Department of \\ Radiology, University of Pennsylvania, Philadelphia, PA 19104; ${ }^{\mathrm{b}}$ Division of Respiratory and Sleep \\ Medicine, Children's Hospital at Montefiore, Bronx, New York, 10467.
}

\begin{abstract}
Mechanisms leading to Obstructive Sleep Apnea Syndrome (OSAS) in obese children are not well understood. We previously analyzed polysomnographic and demographic data to study the anatomical characteristics of the upper airway and body composition in two groups of obese children with and without OSAS, where object volume was evaluated. In this paper, in order to better understand the disease we expand the analysis considering a variety of features that include object-specific features such as size, surface area, sphericity, and image intensity properties of fourteen objects in the vicinity of the upper airway, as well as inter-object relationships such as distance between objects. Our preliminary results indicate several interesting phenomena: volumes and surface areas of adenoid and tonsils increase statistically significantly in OSAS. Standardized T2-weighted MR image intensities differ statistically significantly between the two groups, implying that perhaps intrinsic tissue composition undergoes changes in OSAS. Inter-object distances are significantly different between the two groups for object pairs (skin, oropharynx), (skin, fat pad), (skin, soft palate), (mandible, tongue), (oropharynx, soft palate), (left tonsil, oropharynx), (left tonsil, fat pad) and (left tonsil, right tonsil). We conclude that treatment methods for OSAS such as adenotonsillectomy should respect proportional object size relationships and spatial arrangement of objects as they exist in control subjects.
\end{abstract}

Keywords: Upper Airway, Obstructive Sleep Apnea Syndrome, MR Imaging, Image Analytics

\section{INTRODUCTION}

Obstructive Sleep Apnea Syndrome (OSAS) in obese children is usually associated with other co-morbidities that have impact on cognitive function, behavioral disorders, cardiovascular function, and metabolic function [1]. The most significant risk factor for moderate sleep-disordered breathing in children and adolescents (2-18 years of age) has been associated with obesity (odds ratio of 4.59) [2,3]. The mechanisms and contributing factors for OSAS in obese children are not entirely well understood yet. Image-derived anatomical features such as organ size and volume have been analyzed and the correlation between anatomical features and apnea-hypopnea index (AHI) have been studied $[4,5]$. Currently, medical images used for OSAS quantitative analysis include x-ray computed tomography (CT) [6], optical coherence tomography (OCT) [7], and magnetic resonance imaging (MRI) [2, 4-6, 8]. While OCT offers high spatial and temporal resolution, it is somewhat intrusive, has poor depth of penetration, and has shadowing effects. CT affords good spatial and reasonable temporal resolution but has poor contrast resolution for soft tissue structures and has radiation concerns especially for imaging children. MRI therefore seems to be the modality of choice for studying OSA. Measurements derived from MRI include upper airway cross-sectional area at specific locations [9, 10], upper airway volume / space [11], longitudinal diameter, collapsibility [12], texture of airway muscle [13], and combinations of some of these features [14].

In this paper, we examine a variety of features including object-specific size, surface area, volume, shape, and image intensity properties, as well as inter-object relationships and the correlation among object-specific features. We quantitatively analyze these properties as related to 14 major structures in the upper airway region in normal children and children with OSAS.

\section{METHODS}

Institutional Review Board approval was obtained for this prospective study. The image data sets utilized in this study pertain to thirty subjects involving male and female children (BMI > 95th percentile), 8-17 years of age. All have normal

Medical Imaging 2015: Biomedical Applications in Molecular, Structural, and Functional Imaging, edited by Barjor Gimi, Robert C. Molthen, Proc. of SPIE Vol. 9417, 94172J

(C) 2015 SPIE $\cdot$ CCC code: $1605-7422 / 15 / \$ 18 \cdot$ doi: $10.1117 / 12.2082574$ 
development and intact adenoid and tonsils. Diagnosis of OSAS or non-OSAS is confirmed by polysomnography. 15 subjects are in the OSAS group (13.9 \pm 2.0 years) and another 15 in the control group (13.6 \pm 2.8 years). The MR image data utilized consist of axial T2-weighted and sagittal T1- and T2-weighted sequences with the following acquisition parameters. T2-weighted: TR/TE $=8274.3 / 82.6 \mathrm{msec}$, T1-weighted: TR/TE $=517.7 / 7.6 \mathrm{msec}, 400 \times 400 \times 35-50$, voxel size $0.5 \times 0.5 \times 3.3 \mathrm{~mm}^{3}$.

For consistency, a definition of the neck body region was arrived at in terms of a starting and ending anatomic axial slice locations for every subject. All image data were clipped to match this definition of the body region. In the same way, each of the 14 objects considered in the study was consistently defined regarding its anatomy to specify what aspects of the object are considered for inclusion (see Table 1). Each object was carefully delineated in all 30 subject image sets by using a combination of image segmentation tools [15] under close human supervision. All results were checked for accuracy via 3D surface renditions.

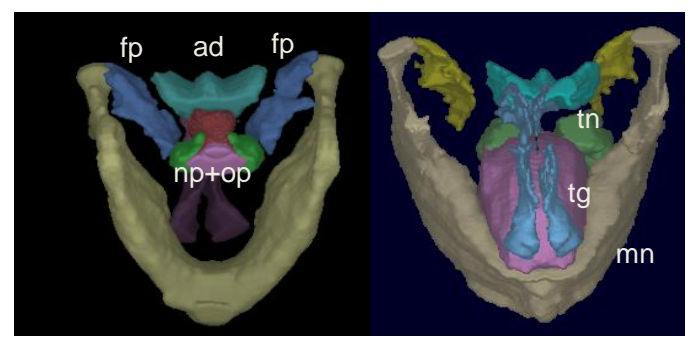

Figure 1.3D surface renditions of some segmented objects from one subject. Objects shown in different combinations are: np (nasopharynx), op (oropharynx), mn (mandible), tn (tonsils), tL \& tR (left and right tonsil), fp (fat pad), ad (adenoid), tg (tongue).

Measurements derived from the object assembly are described in the following sections.

\begin{tabular}{|c|c|c|}
\hline \multicolumn{3}{|c|}{ Table 1. Objects studied in the upper airway region and their abbreviations and definitions. } \\
\hline Object & Abbreviation & Definition of object \\
\hline Skin & sk & $\begin{array}{l}\text { The outer boundary of the neck region skin. The interior region constitutes the entire neck body } \\
\text { region. The superior boundary is defined by the superior aspect of the eyes. The inferior boundary } \\
\text { is defined by } 2 \text { slices }(6.6 \mathrm{~mm}) \text { inferior to the inferior aspect of the mandible. }\end{array}$ \\
\hline Pharynx \& mandible & $\mathrm{pm}$ & Grouping of $\mathrm{mn}$ and $\mathrm{ph}$. \\
\hline Mandible & $\mathrm{mn}$ & The outer boundary of the mandible. \\
\hline Pharynx & $\mathrm{ph}$ & Grouping of np and op. \\
\hline Nasopharynx & $\mathrm{np}$ & $\begin{array}{l}\text { The nasopharynx sub region is defined by the airways, where the inferior boundary is defined by } \\
\text { the inferior aspect of the soft palate. }\end{array}$ \\
\hline Oropharynx & op & $\begin{array}{l}\text { The pharynx sub region is defined by the airways, where the inferior boundary is defined by the } \\
\text { superior aspect of the epiglottis and superior boundary by the inferior aspect of the soft palate. }\end{array}$ \\
\hline Fat pad & fp & The outer boundary of the lateral pharyngeal fat pad. \\
\hline Adenoid \& Tonsil & at & Soft tissue grouping of th and ad. \\
\hline Tonsils & th & Grouping of tR and tL. \\
\hline Right Tonsil & tR & The outer boundary of the right tonsil. \\
\hline Left Tonsil & $\mathrm{tL}$ & The outer boundary of the left tonsil. \\
\hline Tongue & $\operatorname{tg}$ & The outer boundary of the tongue, segmented in the sagittal view. \\
\hline Soft Palate & $\mathrm{sp}$ & The outer boundary of the soft palate, adjacent and posterior to the hard palate. \\
\hline Adenoid & $\mathrm{ad}$ & The outer boundary of the adenoid. \\
\hline
\end{tabular}

Size $\left(S_{\lambda}\right)$ : For each object, we describe its linear size estimate $S_{\lambda}$, as defined by

$$
S_{\lambda}=\sqrt{\left(\lambda_{1}+\lambda_{2}+\lambda_{3}\right)}
$$

where $\lambda_{1}, \lambda_{2}$, and $\lambda_{3}$ are the eigenvalues obtained by Principal Component Analysis of the entire 3D object region. $S_{\lambda}$ described above is an absolute measure. We normalize this measure by the diagonal of a box that encloses the mandible, the idea being that the mandible partially encloses the upper-airway region, constraining other objects, and may be a good indicator of the overall size of a subject. If $L$ denotes this normalizing length for a subject, then the normalized size measures of the objects for the subject were expressed as $S_{\lambda} / L$.

Volume $\left(S_{V}\right)$ : The volume enclosed by the surface is computed for each object. We use the normalized measure $S_{V} / L^{3}$. 
Surface area $\left(S_{A}\right)$ : The surface area of each object is computed from its triangular surface representation. We use the normalized measure $S_{A} / L^{2}$.

Standardized image intensity statistics $\left(H_{M}\right)$ : We used the intensity standardization methods described in [38], which have been shown to achieve tissue-specific numeric meaning to overcome the problem of non-standardness of MR image intensities, to study if tissue-specific properties differed in the two groups of subjects. $H_{M}$ denotes the mean standardized image intensity over all voxels within the object under consideration. The standardized intensity scale was set to the range [0,4095] for all objects and all MRI protocols.

Sphericity $\left(S_{P}\right)$ : The formula given below, which is dependent on volume $S_{V}$ and surface area $S_{A}$ of the object, is derived in such a manner that for a sphere this measure assumes a value of 1 . All other shapes will have higher values. It expresses how an object deviates from being spherical.

$$
S_{P}=\frac{\sqrt[3]{36 \pi S_{V}^{2}}}{S_{A}} .
$$

Inter-object distance $(d(A, B))$ : Object relationships are studied in terms of the distances $d(A, B)$ between objects for every pair of objects. The geometric center of each object is taken to be its reference point, and the distance $d(A, B)$ between any two objects $A$ and $B$ is defined as the Euclidean distance between their geometric centers. The distances were also normalized by $L$ as for size $S_{\lambda}$.

Object size correlation: We calculated the Pearson coefficient of correlation between every pair of objects for $S_{\lambda} / L$.

\section{RESULTS}

Table 2. Mean and standard deviation of $S_{\lambda} / L, S_{V} / L^{3}, S_{A} / L^{2}, S_{P}, H_{M}$, and $d(A, B) / L$ for those objects which showed statistically significant $(\mathrm{p}<0.05)$ differences between the two groups.

\begin{tabular}{|c|c|c|c|c|}
\hline Measure & Objects & Control & OSAS & p-value \\
\hline \multirow{2}{*}{$S_{\lambda} / L$} & $\mathrm{~s}_{\mathrm{k}}$ & $0.43(0.02)$ & $0.44(0.02)$ & 0.05 \\
\hline & $\mathrm{ad}$ & $0.07(0.01)$ & $0.08(0.01)$ & 0.05 \\
\hline \multirow{4}{*}{$S_{V} / L^{3}$} & at & $0.003(0.001)$ & $0.004(0.002)$ & 0.04 \\
\hline & tn & $0.0017(0.0009)$ & $0.0021(0.0008)$ & 0.05 \\
\hline & $\mathrm{tL}$ & $0.0008(0.0004)$ & $0.0010(0.0004)$ & 0.05 \\
\hline & $\mathrm{tR}$ & $0.0009(0.0005)$ & $0.0011(0.0004)$ & 0.05 \\
\hline \multirow{7}{*}{$S_{A} / L^{2}$} & sk & $5.09(0.43)$ & $5.51(0.58)$ & 0.04 \\
\hline & tn & $0.15(0.05)$ & $0.17(0.04)$ & 0.05 \\
\hline & $\mathrm{tL}$ & $0.07(0.02)$ & $0.08(0.02)$ & 0.03 \\
\hline & $\mathrm{tR}$ & $0.08(0.03)$ & $0.09(0.02)$ & 0.03 \\
\hline & at & $0.29(0.07)$ & $0.34(0.07)$ & 0.02 \\
\hline & $\mathrm{fp}$ & $0.23(0.04)$ & $0.28(0.07)$ & 0.02 \\
\hline & $\mathrm{ad}$ & $0.14(0.04)$ & $0.17(0.05)$ & 0.03 \\
\hline \multirow{2}{*}{$S_{P}$} & $\mathrm{tR}$ & $0.49(0.06)$ & $0.45(0.05)$ & 0.01 \\
\hline & $\mathrm{ad}$ & $0.30(0.02)$ & $0.28(0.02)$ & 0.001 \\
\hline \multirow{4}{*}{$H_{M}$} & sk & $1233.50(40.20)$ & $1269.90(42.00)$ & 0.01 \\
\hline & $\mathrm{ph}$ & $297.60(42.40)$ & $261.50(44.80)$ & 0.02 \\
\hline & $\mathrm{fp}$ & $2423.90(139.80)$ & $2352.90(81.70)$ & 0.05 \\
\hline & $\mathrm{np}$ & $328.60(56.90)$ & $278.90(56.60)$ & 0.01 \\
\hline \multirow{8}{*}{$d(A, B) / L$} & sk, op & $0.18(0.04)$ & $0.21(0.04)$ & 0.02 \\
\hline & sk,fp & $0.09(0.03)$ & $0.12(0.05)$ & 0.03 \\
\hline & sk,sp & $0.21(0.03)$ & $0.23(0.05)$ & 0.04 \\
\hline & $\mathrm{mn}, \operatorname{tg}$ & $0.02(0.01)$ & $0.04(0.03)$ & 0.04 \\
\hline & op-tL & $0.09(0.01)$ & $0.08(0.01)$ & 0.004 \\
\hline & op-sp & $0.11(0.03)$ & $0.12(0.02)$ & 0.05 \\
\hline & fp-tL & $0.16(0.02)$ & $0.12(0.02)$ & 0.05 \\
\hline & tL-tR & $0.15(0.03)$ & $0.13(0.02)$ & 0.05 \\
\hline
\end{tabular}


In Table 2, mean and standard deviation of size, surface area, volume, sphericity, mean standardized image intensity within the object region, and distance $d(A, B) / L$ are presented for both control and OSAS groups for those among 14 objects which showed significant $(\mathrm{p}<0.05)$ differences between the two groups. In Figure 2 , we display the heat maps of size $\left(S_{\lambda}\right)$ correlations for the two groups. The corresponding p-value maps of statistical significance are displayed below the heat maps.

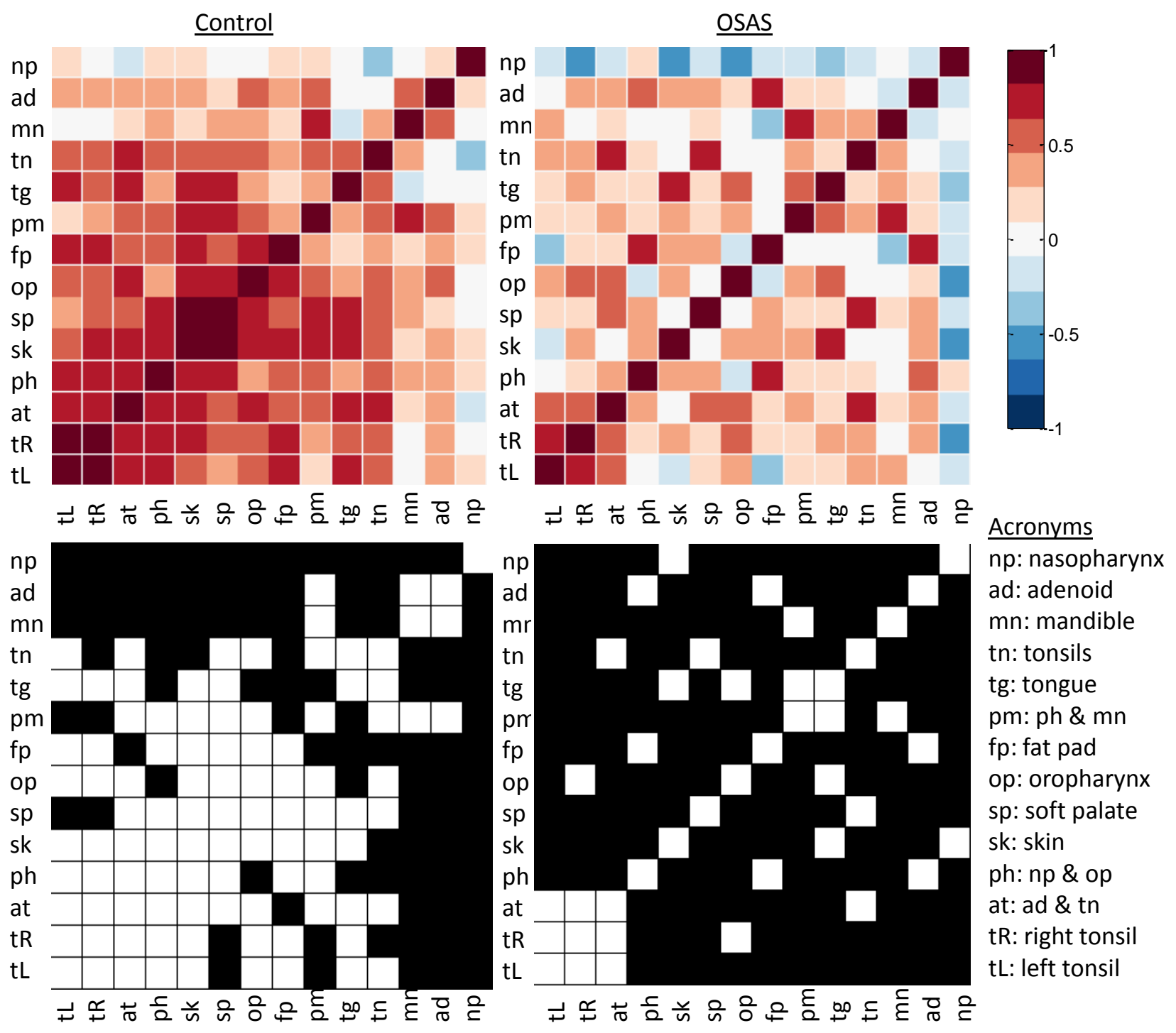

Figure 2. Coefficients of correlation among objects depicted via heat maps for size measure $S_{\lambda} / L$. Left: heat map for control group. Right: Heat map for OSAS group. The corresponding p-value maps are also displayed below the heat maps, where $\mathrm{p}<0.05$ is displayed in white.

\section{DISCUSSION AND CONCLUSIONS}

Differences were observed in the distribution of volume between the control and OSAS groups for at (adenoid and tonsils combined). There was statistically significant difference in size and sphericity of ad (adenoid) and also in surface area of at (adenoid and tonsils), fp (fat pad), and ad (adenoid) between the two groups. Object volume based comparisons were previously studied by others with similar observations [5]. The main difference in our study was that we normalized all geometric measurements with respect to $L$, the linear size of the mandible. Interestingly, there were several objects with statistically significant differences in standardized T2-weighted image intensity: sk (entire body 
region within skin boundary), ph (pharynx), fp (fat pad), and np (nasopharynx), perhaps implying changes in the object tissues in OSAS. For fat pad, pharynx, and nasopharynx, mean standardized intensity in OSAS was lower than in the control group. Distances between objects increased statistically significantly in the OSAS group compared to the control group for object pairs (skin, oropharynx), (skin, fat pad), (skin, soft palate), (mandible, tongue), and (oropharynx, soft palate), while it decreased for object pairs (oropharynx, left tonsil), (fat pad, left tonsil), and (left tonsil, right tonsil).

We observe that the heat maps are overall remarkably different between the two groups. The objects in the control group are largely positively correlated in size while those in the OSAS group have predominantly weak positive correlations, most of which are not statistically significant. This implies that object size proportional relationships which are maintained in the control group are lost in the patient group. An intriguing change in correlation can be observed between fat pad and (left and right) tonsils; in the OSAS group these objects become uncorrelated. Another interesting behavior is the correlation between adenoid and fat pad size, which is enhanced for the OSAS group, and so also for pharynx and fat pad.

A limitation of our study is the small number of data sets employed in each group. However, the data provided preliminary results for a novel way of analyzing image data in OSAS where the entire anatomy of the region is considered for the purpose of understanding what relations among objects may be important in describing the OSAS phenomenon, which have not been discussed in previous publications. As we gather more data, the analysis will be extended and the results will be updated. We intentionally analyzed parameters individually to study what measures may be important in distinguishing between the two groups. Such an approach is inevitable as a first step of selecting a small set of key parameters which may be subsequently utilized in a composite manner in a larger clinical study focusing on specific clinical questions.

The most important observations from our analysis may be summarized as follows: (1) Standardized T2-weighted image intensities differ statistically significantly between the two groups for some objects (entire body region, fat pad, pharynx, and nasopharynx). (2) Volumes and surface areas of adenoid and tonsils increase statistically significantly in OSAS, as has been found in previous studies. (3) Distances between objects increase in the OSAS group for object pairs (skin, oropharynx), (skin, fat pad), (skin, soft palate), (mandible, tongue), and (oropharynx, soft palate), while they decrease for object pairs (left tonsil, oropharynx), (left tonsil, fat pad), and (left tonsil, right tonsil). This method of analysis may lead to a better understanding of the mechanisms underlying OSAS. For example, changes in standardized T2-weighted image intensities in objects may imply that intrinsic tissue composition undergoes changes in OSAS. The results on object sizes and distances imply that treatment methods should respect the size relationships that exist among objects. Treatment methods should also respect normal spacing among objects.

\section{Acknowledgements}

The research reported here is supported by a DHHS grant HL105212.

\section{REFERENCES}

[1] H. Muzumdar and R. Arens, "Physiological effects of obstructive sleep apnea syndrome in childhood," Respir Physiol Neurobiol, vol. 188, 370-382 (2013).

[2] S. L. Davidson Ward, R. Amin, R. Arens, C. Zhongping, S. Davis, E. Gutmark, et al., "Pediatric sleep-related breathing disorders: advances in imaging and computational modeling," IEEE Pulse, vol. 5, 33-39 (2014).

[3] R. Arens and H. Muzumdar, "Childhood obesity and obstructive sleep apnea syndrome," J Appl Physiol (1985), vol. $108,436-444$ (2010).

[4] K. Nandalike, K. Shifteh, S. Sin, T. Strauss, A. Stakofsky, N. Gonik, et al., "Adenotonsillectomy in obese children with obstructive sleep apnea syndrome: magnetic resonance imaging findings and considerations," Sleep, vol. 36, 841-847 (2013).

[5] R. Arens, S. Sin, K. Nandalike, J. Rieder, U. I. Khan, K. Freeman, et al., "Upper airway structure and body fat composition in obese children with obstructive sleep apnea syndrome," Am J Respir Crit Care Med, vol. 183, 782 787 (2011).

[6] S. Li, H. Shi, X. Dong, S. Qu, W. Dong, and G. Wang, "Quantitative evaluation of the upper airway by CT scan with the Muller maneuver," Lin Chuang Er Bi Yan Hou Ke Za Zhi, vol. 19, 104-107 (2005). 
[7] K. Wijesundara, C. Zdanski, J. Kimbell, H. Price, N. Iftimia, and A. L. Oldenburg, "Quantitative upper airway endoscopy with swept-source anatomical optical coherence tomography," Biomed Opt Express, vol. 5, 788-799 (2014).

[8] R. Arens, J. M. McDonough, A. M. Corbin, M. E. Hernandez, G. Maislin, R. J. Schwab, et al., "Linear dimensions of the upper airway structure during development: assessment by magnetic resonance imaging," Am J Respir Crit Care Med, vol. 165, 117-122 (2002).

[9] R. Arens, J. M. McDonough, A. M. Corbin, N. K. Rubin, M. E. Carroll, A. I. Pack, et al., "Upper airway size analysis by magnetic resonance imaging of children with obstructive sleep apnea syndrome," Am J Respir Crit Care Med, vol. $167,65-70$ (2003).

[10] S. A. Nouraei, D. W. McPartlin, S. M. Nouraei, A. Patel, C. Ferguson, D. J. Howard, et al., "Objective sizing of upper airway stenosis: a quantitative endoscopic approach," Laryngoscope, vol. 116, $12-17$ (2006).

[11] S. C. Pereira, R. T. Beltrao, G. Janson, and D. G. Garib, "Lateral cephalometric radiograph versus lateral nasopharyngeal radiograph for quantitative evaluation of nasopharyngeal airway space," Dental Press J Orthod, vol. 19, 89-93 (2014).

[12] P. P. Hsu, A. K. Tan, E. C. Gan, Y. H. Chan, M. M. Goh, P. K. Lu, et al., "Computer-assisted quantitative upper airway analysis following modified uvulopalatal flap and lateral pharyngoplasty for obstructive sleep apnoea: a prospective case-controlled study," Clin Otolaryngol, vol. 37, 188-196 (2012).

[13] P. Kolhi, J. Jarnstedt, M. Sikio, J. Viik, P. Dastidar, T. Peltomaki, et al., "A texture analysis method for MR images of airway dilator muscles: a feasibility study," Dentomaxillofac Radiol, vol. 43, 1-7 (2014). DOI: 10.1259/dmfr.20130403.

[14] Z. Abramson, S. Susarla, M. August, M. Troulis, and L. Kaban, "Three-dimensional computed tomographic analysis of airway anatomy in patients with obstructive sleep apnea," J Oral Maxillofac Surg, vol. 68, 354-362 (2010).

[15] G. Grevera, J.K. Udupa, D. Odhner, Y. Zhuge, A. Souza, T. Iwanaga, and S. Mishra, "CAVASS: A computer-assisted visualization and analysis software system,” Journal of Digital Imaging, vol. 20(Supplement 1), 101-118 (2007). 